\title{
Simulation and Test Study on Dynamic Characteristic of Air Spring with Auxiliary Chamber
}

\author{
Li-qing Sun ${ }^{1}$, Zhong-xing Li ${ }^{1}$, Xu-feng Shen ${ }^{1}$, Jia-yi Zhu ${ }^{1}$ \\ (School of Auto And Traffic Egineering Jiangsu University Zhengjinag 212013) \\ slq@ujs.edu.cn, Izx@ ujs.edu.cn, sxf@ujs.edu.cn, zhujy@ujs.edu.cn
}

Keywords: air spring auxiliary chamber connecting pipe finite element analysis

\begin{abstract}
In order to improve dynamic characteristics of air spring with auxiliary chamber, finite element model of air spring R1A390-295 with auxiliary chamber connected with pipe is established,and through analysis to the dynamic characteristics of the model, influence discipline of sitffness characteristics to air spring with different pipe, different auxiliary chamber or different initial pressure are analysed under different excitation. The result show that:minor dynamic sitffness is obtained by using larger pipe or under lower excitation frequency,and as volume of auxiliary chamber increases, the spring dynamic sitffness will decrease accordingly and its amplitude tends to gentle,and influence for decreasing the spring dynamic sitffness is not obvious by continuing to increase the auxiliary chamber volume; the spring dynamic sitffness will increases as initial pressure increases. The validity of Finite element model is verified through dynamic characteristic test .
\end{abstract}

\section{Introduction}

The volume of the air spring affects the air suspension stiffness significantly. To adapt to complex condition, auxiliary chamber is added to air spring with Connecting pipe, changes in stiffness of air spring and high suspension performance can be obtained by changing the effective working volume of air spring through adjusting volume of auxiliary chamber .

Air spring system with auxiliary chamber is mainly composed by air spring (main chamber) , connecting pipe and auxiliary chamber, as shown in Fig. 1. The auxiliary chamber increases the total volume of the air spring, so that the stiffness and the vibration frequency of the spring can be reduced.

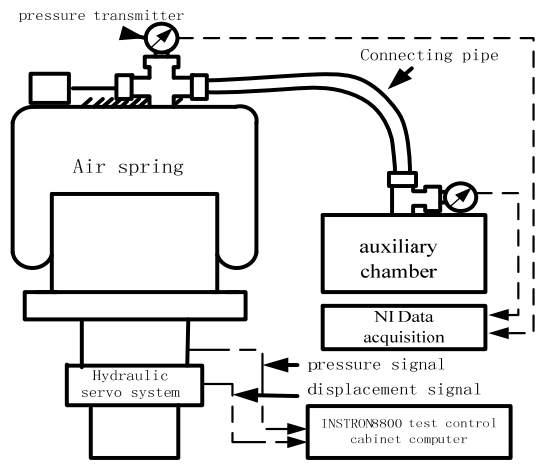

Fig.1 Schematic diagram of air spring with auxiliary chamber

In general research, mathematical model is used to analyze the elastic properties of air spring ${ }^{[1]}$, but its characteristic data can only be obtained by means of physical tests. Additionally, because there are too many assumptions throughout the design process, the accuracy and reliability can not be guaranteed $^{[2-4]}$. Currently, we generally look air spring without auxiliary chamber as object to make finite element analysis.Air in the airbag can not be exchanged with the outside or the other container in the process of vibration. Method of ABAQUS finite element analysis and test is used to obtain the data of dynamic characteristic of air spring with auxiliary chamber in this paper,meanwhile, influence discipline of sitffness characteristics to air spring with different pipe, different auxiliary chamber or different initial pressure are analysed under different excitation.

By Fund: National Natural Science Foundation (51075190) 


\section{Finite element analysis}

Based on Firestone 1R1A390-295 free diaphragm air spring, dynamic characteristics of air spring with auxiliary connected by connecting pipe is analyzed through ABAQUS.

1.1 choosing the element

The finite element model of air spring with auxiliary is composed by five parts: main airbag chamber model, cover model, below piston model, connecting pipe model and auxiliary chamber model . A four-node shell element (defined as S4R in ABAQUS) is adopted in this analysis. Firestone 1R1A 390-295 type air spring of the actual balloon wall thickness of 4mm. Use the rebar element to simulate the cord layer with two layers of 3mm's distance and cord angle of 55 degree.

When it comes to the problem of simulating the gas in the spring, assume that the cavity of the gas is an ideal gas, using ABAQUS hydrostatic fluid unit, so that deformation of the structure and the pressure forced on the boundary fluid can couple mutually.

In such process, two gas elements are adopted: Three-dimensiona and three-node (F3D3) ,and three-dimensional four-node (F3D4) fluid unit.One is the main airbag, in which F3D4 fluid element shares node with the skin shell elements. The upper gas chamber is closed by the F3D3 which is shared by the upper rabbet and the down rabbet. The other is the auxiliary chamber. F3D4 fluid element shares the node with the sidewall rigid element of the chamber. The gas is closed by the three-dimensional three-node on the top and bottom of the chamber. the rigid surfaces of the upper cover and the lower piston are obtained by rotating. The reference node if defined to be on the symmetry axis. Using the rigid body to simulate is allowed because there is no displacement of the auxiliary chamber in the vibration and the chamber is made of metal. Connecting pipe using Flink element as the fluid element to simulate, which is a two-dimensional two-node element F2D2.

1.2 Meshing

The airbag is meshed in the modeling process firstly,then aliquots the node into forty four copies around the symmetry axis, and the node of airbag can be determined. The airbag is connected end to end to every four nodes to form a quadrilateral element, and all quadrilateral elements combine into a finite element model of airbag grid. The gas in the chamber shares nodes with airbag and every four nodes form a gas unit with the reference point on the symmetry axis. Define a reference point in the centre of the upper and lower ending mouth respectively. The reference point forms a closed triangle with the node on the boundary. Similarly, the gas in the auxiliary chamber s is also studied in this way. In order to avoid non-converge and penetration of a rigid body, the grid must be refined appropriately when it comes to passive body airbag ${ }^{[5]}$. Finally, through the completion of the preparation of the INP program the gas unit applied and the final shape of finite element model are achieved, grid of finite element model of the airbag with auxiliary chamber is shown in Fig. 2.

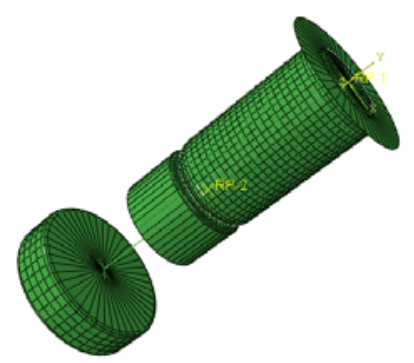

Fig.2 ,Finite element model of air spring with auxiliary chamber

1.3 boundary condition

A multi-point constraint MPC is introduced to make different parts of air spring as a single entity. TIE function is adopted to connect and fix up a circle of boundary nodes of airbag's upper rabbet with the contact nodes of upper cover ${ }^{[6]}$. The fluid unit of upper airbag are connected with the reference points of upper cover. The TIE function of lower piston is defined similarly as that of upper cover.

\section{Model validation}

2.1 Simulation validation 
Initial air of $0.1 \mathrm{MPa} 0.15 \mathrm{MPa} 0.2 \mathrm{MPa}$ and $0.25 \mathrm{Mpa}$ is filled in air spring successively, and frequency range of sinusoidal excitation that exert on the air spring is $0.5 \sim 10 \mathrm{~Hz}$, and the amplitude is $10 \mathrm{~mm}$;connecting pipe:pipe length $0.8 \mathrm{~m}$, pipe diameter $0 \mathrm{~mm}, 6 \mathrm{~mm}, 8 \mathrm{~mm}, 12 \mathrm{~mm}, 16 \mathrm{~mm}$ and $20 \mathrm{~mm}$; volume of auxiliary chamber is $5 \mathrm{~L}, 10 \mathrm{~L}$ and $15 \mathrm{~L}$; curve of dynamic stiffness to air spring is obtained under different frequency by finite element simulation.

2.2 Dynamic characteristic test

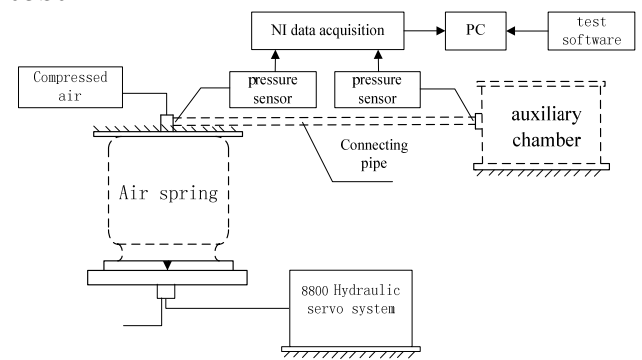

Fig. 3. test bench system

According to the technical requirements of GB / T 13061-91, dynamic characteristic test of Firestone1R1A390-295 air spring with auxiliary chamber is carried out on theINSTRON8800 electro-hydraulic servo vibration test bench, test bench system is shown in Fig 3.

Fig. 4 shows influence to dynamic stiffness with different pipe under different frequency,and initial pressure is $0.15 \mathrm{Mpa}$, and volume of auxiliary chamber is $10 \mathrm{~L}$.

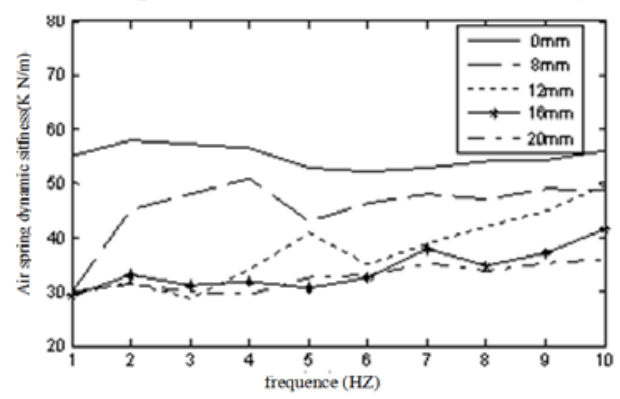

(a) curve of simulation

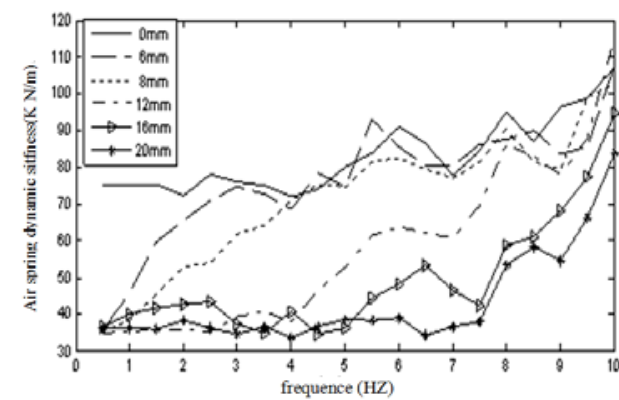

(b) curve of test

Fig 4. influence of pipe diameter's on the dynamic stiffness of the air spring .

In Fig.4, the dynamic stiffness of air spring with auxiliary chamber increases as the excitation frequency increases, within a frequency range, changes rapidly. In this Fig, stiffness curve corresponding with diameter of $6 \mathrm{~mm}, 8 \mathrm{~mm}$ can soon reach a higher value at low frequencies. the value of dynamic stiffness of the air spring is closer to the auxiliary chamber air spring with a smaller pipe diameter and higher excitation frequency .on the other hand ,with a larger diameter, and the lower frequency, in is more conducive to the flow of gas and the rigidity of the air spring is smaller.

Fig. 5 shows influence to dynamic stiffness with different auxiliary chamber under different frequency, , and initial pressure is $0.15 \mathrm{MPa}$, and pipe diameter is $16 \mathrm{~mm}$.

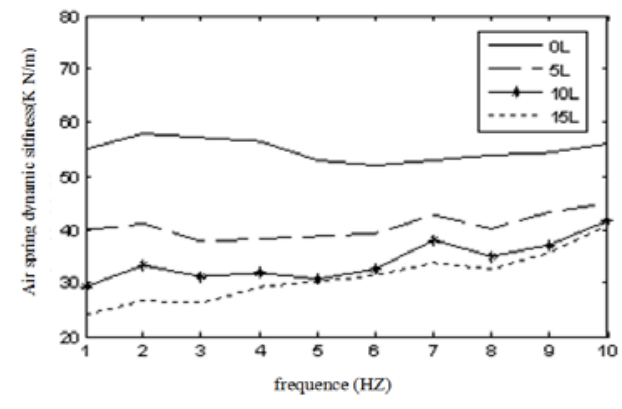

(a) curve of simulation

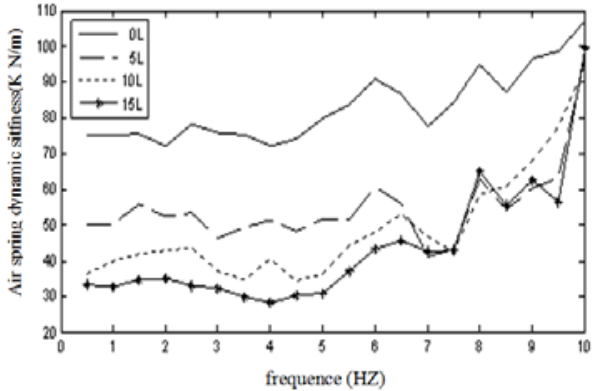

(b) curve of test

Fig. 5 influence to dynamic stiffness with auxiliary chamber volume

In Fig.5, dynamic stiffness is similar to non- auxiliary chamber. Meanwhile, dynamic stiffness of the air spring is gradually reduced when volume of auxiliary chamber increases, but the amplitude of the reduction, the magnitude of change is greater between the volume change in the interval of 0 to $5 \mathrm{~L}$ than 5Lto 10L or 10L to 15L. as volume of auxiliary chamber increases, the spring dynamic sitffness 
amplitude tend to gentle,and influence for decreasing the spring dynamic sitffness is not obvious by continuing to increase the auxiliary chamber volume.

Fig.6 shows influence to dynamic stiffness with different initial air pressure under different frequency, ,and volume of auxiliary chamber is10L,and pipe diameter is $12 \mathrm{~mm}$.

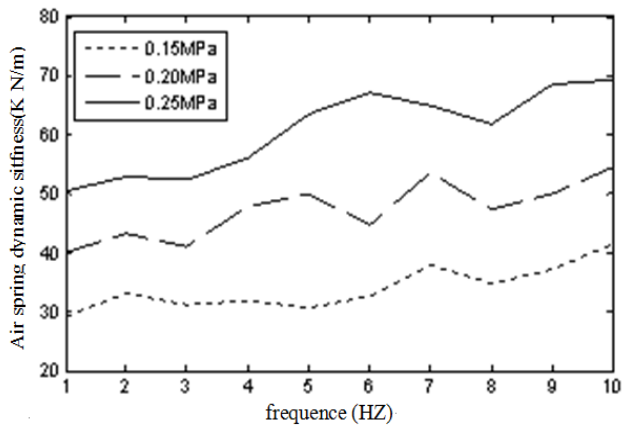

(a) curve of simulation

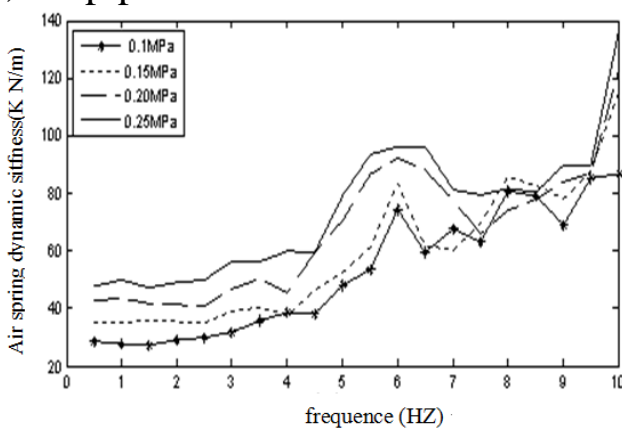

(b) curve of test

Fig.6 influence of different initial pressure on the dynamic stiffness of the air spring

In Fig 6, in the entire frequency range of the interval, the dynamic stiffness of the air spring increases when the initial pressure increases, because at the same height, the higher the pressure is, the greater the gas concentration is, the greater the force between the gas molecules is, the greater force is necessary to compress the gas. i.e., the stiffness of the air spring is greater.

The above simulation results consistent with the trend of experimental curve, but the Test curve of dynamic stiffness is of the fluctuations at high frequencies, because in the actual working conditions; high-frequency excitation makes the movement of gas in the gas more complex, causing irregular drastic changes of dynamic stiffness .However, in the analysis of finite element, the gas hydrostatic fluid unit selected as gas in the airbag is in slight difference from the actual gas in the airbag during the movement. The gas flow is relatively stable.

\section{Conclusions}

(1) The finite element model of air spring with auxiliary chamber is established on finite element method. The calculation of its dynamic characteristics and the characteristics test showed high consistence. The validity of Finite element model is verified through characteristic test .

(2) Minor dynamic sitffness is obtained by using larger pipe or under lower excitation frequency,and as volume of auxiliary chamber increases, dynamic sitffness will decrease accordingly and influence for decreasing the spring dynamic sitffness is not obvious by continuing to increase the auxiliary chamber volume; the spring dynamic sitffness will increases as initial pressure increases.

\section{References}

[1] Li Rui..The Finite Element Analysis for Static and Dynamic Characteristics of the Automotive Air Spring[D].Shang Hai:Dong Hua,2009.

[2] Mats Berg.A Three Dimension Air Spring Model with Friction and Orifice Damping[J]. Vehicle System Dynamics, 1999,33 (Supplement):528-539.

[3] Tu DeXin, Hang ChangWen, etc.. Study on the Influence of Cord Parameters on the Vertical Stiffness of Air Springs for vehicles based on ABAQUS [J]. Auotmobile Technology, 2011(2):10-17.

[4] Yuan Chunyuan, Zhou Kongkang, Wang Guolin, et al. Simulation and experimental investigation of mechanical property of air spring for vehicles [J]. Transactions of the Chinese Society of Agricultural Engineering,, 2008, 24(3):38-42.

[5] Wang Huai 1, GE Ru hai 1, LI Yan li, etc. The Influence of Meshing Density on Automobile Crashing Simulation and the Efficiency of Calculation.[J].ZhenJiang: ournal of Jiangsu University of Science and Technology, , 2002,23(4):29-32.

[6] ABAQUS Keywords Reference Manual 6.10-1.HKS Co.Ltd. 